\title{
Essais
}

ESSAIS

Revue interdisciplinaire d'Humanités

14 | 2018

Plurilinguismes en construction

\section{Promouvoir l'apprentissage des langues en ÉcosseExemple d'un projet scolaire qui valorise les identités plurilingues}

Malika Pedley

\section{(2) OpenEdition \\ Journals}

Édition électronique

URL : http://journals.openedition.org/essais/346

DOI : $10.4000 /$ essais.346

ISSN : 2276-0970

Éditeur

École doctorale Montaigne Humanités

\section{Édition imprimée}

Date de publication : 1 juin 2018

Pagination : 117-133

ISBN : 979-10-97024-06-2

ISSN : $2417-4211$

Référence électronique

Malika Pedley, «Promouvoir l'apprentissage des langues en ÉcosseExemple d'un projet scolaire qui valorise les identités plurilingues », Essais [En ligne], 14 | 2018, mis en ligne le 01 décembre 2019, consulté le 12 décembre 2019. URL : http://journals.openedition.org/essais/346 ; DOI : 10.4000/ essais.346 


\section{Promouvoir l'apprentissage des langues en Écosse Exemple d'un projet scolaire qui valorise les identités plurilingues}

\section{Malika Pedley}

La situation linguistique du Royaume-Uni est marquée par une dichotomie : face à un multilinguisme qui se justifie par la présence de nombreuses langues allochtones et par la protection de langues autochtones, le monolinguisme de l'anglais reste un fait incontestable et assumé pour une grande partie de la société britannique. L'apprentissage des langues en milieu scolaire restant marginal témoigne entre autres d'un manque de motivation à maîtriser des langues autres que l'anglais. Des politiques linguistiques éducatives en adéquation avec les objectifs de plurilinguisme européen sont mises en œuvre pour tenter de contrer cette tendance.

Cette double réalité linguistique constitue le fil conducteur de notre article : dans un premier temps, nous nous poserons la question suivante : dans une telle situation, comment et par quels moyens peut-on promouvoir l'apprentissage des langues à l'école ? De cette question découleront les suivantes : comment cette dynamique de promotion de l'apprentissage des langues peut-elle nous renseigner sur le multilinguisme en tant que système au Royaume-Uni ? Comment les représentations vis-à-vis du multilinguisme évoluent-elles avec cette dynamique?

Pour comprendre cette problématique, il convient de préciser le cadre de recherche dans lequel elle s'inscrit : la recherche doctorale dont il est question ici a pour objet d'étude le multilinguisme au Royaume-Uni, qu'elle entend considérer comme un système global et participatif; en d'autres termes, plutôt que de caractériser le multilinguisme de cet État en distinguant des catégories de langues, l'objectif est davantage de le voir comme un tout, afin de démontrer que sans faire abstraction de la diversité des situations qui caractérisent les langues en présence, il peut y avoir suffisamment de points communs entre celles-ci pour considérer le multilinguisme, comme un ensemble hétérogène mais cohérent qu'il convient d'exploiter comme une ressource culturelle, économique et linguistique pour la société. 
Ainsi, après avoir présenté les caractéristiques linguistiques et politiques du Royaume-Uni, nous nous intéresserons à la stratégie pour promouvoir l'apprentissage des langues qui consiste à faire du multilinguisme latent une ressource ; dans cette deuxième partie, nous prendrons le temps de présenter le projet Mother Tongue Other Tongue, qui relève de cette stratégie et qui constitue l'enquête de terrain de la recherche doctorale dont il est question ici. Enfin, nous présenterons nos objectifs de recherche vis-à-vis de ce projet semi-scolaire en troisième partie, à savoir étudier son déroulement pour comprendre le multilinguisme tel qu'il est perçu par des enfants plurilingues.

\section{Le Royaume-Uni : situation linguistique et politique}

Six langues sont aujourd'hui reconnues langues régionales ou minoritaires au Royaume-Uni selon la charte du Conseil de l'Europe ${ }^{1}$ : le gallois au Pays de Galles, le gaélique écossais et le scots en Écosse, l'irlandais et l'ulster-scots en Irlande du Nord et le cornique en Cornouailles (comté d'Angleterre). Sur ces territoires, les pratiques linguistiques restent minoritaires mais l'enseignement de ou en langue régionale ou minoritaire est en pleine expansion (Conseil de l'Europe, 2014). Le gallois est la langue qui présente la vitalité la plus prometteuse, avec $19 \%$ de la population galloise qui se déclare capable de parler cette langue en $2011^{2}$. Deux de ces langues ont un statut d'officialité : le gallois au Pays de Galles et le gaélique écossais en Écosse. Toutes ces langues ne bénéficient cependant pas du même degré de protection et de promotion par la charte. À l'heure actuelle, d'autres parlers comme le geordie dans le Nord de l'Angleterre revendiquent une certaine légitimité mais n'ont pas été reconnus en tant que langues. Les langues régionales ou minoritaires seraient donc les seules langues autochtones mis à part l'anglais toujours présentes au Royaume-Uni.

Outre ces langues, des centaines de langues allochtones sont parlées à travers le Royaume-Uni. Depuis 2011, conséquence de la vague migratoire des années 2000, le polonais est unanimement la première langue parlée après l'anglais. Viennent ensuite les langues du sous-continent indien (panjabi, ourdou) jusqu'en 2011 première langue après l'anglais (bengali, gujarati) pour l'Angleterre et le Pays de Galles ainsi que pour l'Écosse, puis l'arabe, le français et les langues dites « chinoises» (cantonais, mandarin...) $)^{3}$.

1 La Charte inclut le mannois dans la liste de langues du Royaume-Uni. Or, l'île de Man est politiquement indépendante du Royaume-Uni mais toujours sous la souveraineté britannique. Ici, puisque nous traitons exclusivement du Royaume-Uni, nous nous permettons d'évincer le cas de cette langue. CONSEIL DE L'EUROPE, Third report of the Committee of Experts in respect of the United Kingdom, European Charter for Regional or Minority Languages, $4^{\text {th }}$ monitoring cycle, 2014, https://www.coe.int/t/dg4/education/minlang/Report/EvaluationReports/UKECRML4_ en.pdf, $22 / 10 / 2015$.

2 OFFICE FOR NATIONAL STATISTICS, http://www.ons.gov.uk/ons/dcp171778_297002.pdf, 2013, 22/10/2015.

3 Pour plus de détails, consulter les publications respectives concernant les recensements de 
La diversité linguistique et culturelle qui marque le Royaume-Uni aujourd'hui est en fait une situation tout à fait contemporaine. Certains évènements du siècle passé permettent de comprendre l'évolution sociolinguistique et politique de cet État.

La pratique de langues allochtones sur le territoire britannique s'explique par différentes vagues migratoires durant le $\mathrm{XX}^{\mathrm{e}}$ siècle, conséquentes d'un besoin de renouvellement de population au lendemain de la Seconde Guerre Mondiale, de conditions d'accès au sol britannique facilité pour les «citoyens du Commonwealth » lors de la chute de l'Empire colonial, et de politiques d'immigration plus ou moins accueillantes selon les périodes successives ${ }^{4}$.

La chute de l'Empire colonial a également eu des conséquences sur le sentiment d'appartenance nationale des Britanniques. En effet, si la construction de l'Empire a favorisé un certain nationalisme britannique au XVIII ${ }^{e}$ siècle fédérant gallois, écossais ou anglais, la désillusion conséquente de l'échec de ce projet a conduit à un repli identitaire. L'émancipation des divers peuples constitutifs du Royaume-Uni s'est accompagnée de revendications linguistiques, culturelles, identitaires et politiques ${ }^{5}$.

Le Royaume-Uni a signé et ratifié la Convention Cadre pour la Protection des Minorités Nationales du Conseil de l'Europe ${ }^{6}$ et reconnait ainsi en son sein l'existence de minorités nationales, dont la définition reprend celle de "groupe racial " ("racial group») : " groupe de personnes défini par la couleur, la race, la nationalité (y compris la citoyenneté) ou les origines ethniques ou nationales ${ }^{7}$. En ce sens, les langues parlées par les minorités (dont font partie par exemple les Gallois et depuis 2014 les Cornouaillais, mais aussi les communautés dites "visibles " comme les Antillais, les Pakistanais ou les Indiens) doivent être protégées en tant que langues de minorités nationales.

population de 2011 : Office for National Statistics, 2013; National Records of Scotland, 2013, http://www.scotlandscensus.gov.uk/documents/censusresults/release2a/StatsBulletin2A.pdf, 22/10/2015 ; Northern Ireland Statistics \& Research Agency, 2013, http://www.nisra.gov.uk/ census/detailedcharacteristics_stats_bulletin_2_2011.pdf, 22/10/2015.

4 Vincent Latour, Le Royaume-Uni et la France au test de l'immigration et à l'épreuve de l'intégration : 1930-2012, Presses Universitaires de Bordeaux, Bordeaux, 2014.

5 Anne Judge, "Linguistiques politiques, langues collatérales et langues différenciées dans le cadre du Royaume-Uni ", in Jean-Michel Éloy(éd.), Des langues collatérales : problèmes linguistiques, sociolinguistiques et glottopolitiques de la proximité linguistique, L'Harmattan, Paris, 2004, p. $145-160$.

6 CONSEIL DE L'EUROPE, Rapport soumis par le Royaume-Uni en vertu de l'article 25, paragraphe 1 de la Convention-cadre pour la protection des minorités nationales, 1999, https://www.coe.int/t/ dghl/monitoring/minorities/3_fcnmdocs/PDF_1st_SR_UK_fr.pdf, 22/10/2015.

7 Notion définie en premier lieu dans le texte Race Relations Act, 1976 (dernière édition) luttant contre la discrimination raciale au Royaume-Uni. Définition extraite de la version française du texte du Conseil de l'Europe (1999). Race Relations Act, 1976, http://www.legislation.gov.uk/ ukpga/1976/74, 22/10/2015. 
Ainsi, il semble maintenant évident que, malgré des divergences quant à leurs origines et quant aux rapports entretenus avec le territoire britannique, la vitalité de ces langues autres que l'anglais est étroitement liée.

D'un point de vue politique, le Royaume-Uni est aujourd'hui un État formé de quatre nations (l'Angleterre, l'Écosse, le Pays de Galles et l'Irlande du Nord) dont chacune dispose depuis la dévolution du pouvoir de l'État en 1998 d'une certaine autonomie politique notamment en ce qui concerne l'éducation et les questions linguistiques. Chacune des nations possède son propre système scolaire (avec non équivalence entre les niveaux et différents examens terminaux). Chaque nation gère aussi ses propres recensements de population ${ }^{8}$.

Les quatre nations sont marquées par certains traits communs comme la suprématie de l'anglais et l'ordre des langues allochtones les plus parlées. En revanche, l'éventuelle présence de langue(s) régionale(s) ou minoritaire(s) et la proportion variable d'individus plurilingues ou pour qui l'anglais n'est pas la langue première confèrent à chacune des nations sa propre identité culturelle et linguistique. Ainsi, si l'ordre des langues allochtones les plus importantes semble le même d'une nation à l'autre, la pluralité linguistique ne prend pas les mêmes proportions : en Écosse, 4,3\% des écoliers utilisent une langue autre que l'anglais à la maison ; en Angleterre, 16,6 \% (école primaire) et $12,5 \%$ (école secondaire) des enfants disposent d'une autre langue que l'anglais ; et en Irlande du Nord, pour 3,1\% de la population, l'anglais n'est pas la langue principale. ${ }^{9}$ Notons ici l'écart entre les différentes formulations des résultats (traduites par nos soins) : les nuances qu'elles présentent en termes de compétences et de pratiques linguistiques, ainsi que les différentes tranches d'âge évoquées rendent encore plus difficile la comparaison entre nations.

À travers les quatre nations, une inadéquation demeure entre un multilinguisme avéré et un enseignement des langues en berne dans l'éducation. Afin d'inverser cette tendance et conformément aux objectifs de plurilinguisme européen, différentes stratégies sont mises en place à travers l'État pour promouvoir l'apprentissage des langues à l'école.

8 Voir note 2.

$9 \quad$ Ibid. 


\section{Faire du multilinguisme latent une ressource pour le plurilinguisme}

\section{Démocratisation des langues dans l'enseignement}

Nuffeld Foundation, organisme qui finance et produit de la recherche en politiques éducatives et sociales, a publié en 2000 le rapport "Languages: The Next Generation " dénonçant l'état déplorable de monolinguisme du Royaume-Uni comme étant en partie responsable d'un affaiblissement économique ${ }^{10}$. Il devient urgent de se tourner vers la richesse multilingue du pays pour gagner en compétitivité mondiale : ce constat présente enfin une opportunité de donner, dans le cadre scolaire, davantage de légitimité et de visibilité aux langues autres que l'anglais parlées au Royaume-Uni ${ }^{11}$.

Il s'agit là d'une proposition qui a fait débat plusieurs fois au RoyaumeUni et qui est ainsi remise au goût du jour. En 1970, le gouvernement publie Bullock Report dans lequel il est entre autres question des langues autres que l'anglais parlées par les communautés ${ }^{12}$ : ce texte annonce l'importance de conserver les langues d'héritage des communautés. Il ne propose cependant aucune mesure pour assurer leur sauvegarde. Ce n'est que quinze ans plus tard, qu'un texte viendra apporter une réponse : Languages for All, plus connu sous le nom de son auteur, Swann Report ${ }^{13}$, déclare que la responsabilité de sauvegarder et d'enseigner ces langues revient aux communautés. En conséquence, les premières "community language schools" voient le jour dans les différents centres urbains du Royaume-Uni. Ces structures de type associatif offrent entre autres un enseignement de langues de familles hors temps scolaire. En réalité, ces structures existaient déjà parmi les communautés dites ethniques minoritaires et dispensaient des enseignements jugés manquants par les communautés à l'école britannique : des cours de religion mais aussi des cours liés aux cultures et traditions du territoire d'origine (musique, chant, danse, etc.). L'ajout de cours de langues dans ces structures associatives a renforcé l'identité de ces communautés autour d'une langue ${ }^{14}$. Une étude faite dans le

10 NUFField FOUNDATION, Languages: The Next Generation, 2000, http://www.nuffieldfoundation.org/sites/default/files/languages_finalreport.pdf, 22/10/2015.

11 Jim Anderson, Charmian Kenner et Eve Gregory, "The National Languages Strategy in the UK: Are Minority Languages Still on the Margins? ", in Christine Helot et Anne-Marie DeMeja (éds), Forging Multilingual Spaces: Integrating Perspectives on Majority and Minority Bilingual Education, Multilingual Matters, Bristol, England, 2008, p. 183-200.

12 Alan Bullock, A language for Life, 1975, http://www.educationengland.org.uk/documents/ bullock/bullock1975.html, 22/10/2015.

13 Lord Swann, Education for All, 1985, http://www.educationengland.org.uk/documents/ swann/swann1985.html, 22/10/2015.

14 Joanna McPake, "Mapping the languages of Edinburgh ", SCOTLANG Seed Project 6, Scottish CILT, 2002, http://www.scilt.org.uk/Portals/24/Library/publications/SCILT_2002_McPake_ Mapping_finalreport.pdf, 22/10/2015, 132 p. 
cadre du projet de recherche "Valuing All Languages in Europe ${ }^{15}$ a recensé 61 langues enseignées dans le cadre de "community language schools" au Royaume-Uni en 2005 (Anderson et al., 2008, p. 194).

Depuis Swann Report, aucun texte d'une telle ampleur n'a été publié. À l'échelle des nations ou bien de localités, on a pu assister à certaines inclusions de langues minoritaires dans le cursus scolaire. Dans l'agglomération de Londres, de 1983 à 1989, des langues minoritaires parlées localement ont pu être enseignées à l'école en tant que matières mais aussi en tant que disciplines non linguistiques (Anderson et al., 2008). Précisons que le caractère multiculturel de Londres est perçu globalement comme un des facteurs conférant à la capitale une place centrale dans le marché économique mondial : Philip Baker et John Eversley ${ }^{16}$ (2000 : p. 71-72) insistent sur cet argument dans leur ouvrage consacré à la diversité linguistique de Londres. On y trouve entre autres un extrait d'un discours prononcé par Tony Blair en 1999 : «London's diversity helps it to compete in today's global market place $»^{17}$.

Aujourd'hui, certaines de ces langues peuvent faire l'objet d'épreuves aux examens GCSEs en Angleterre (équivalent des brevets des collèges) : l'offre dépend des municipalités et reste très rare ${ }^{18}$. Aussi, avec les textes de loi Welsh Language Act ${ }^{19}$ ou Gaelic Language (Scotland) $A c t^{20}$, le gallois au Pays de Galles et le gaélique écossais en Écosse peuvent ou sont aujourd'hui enseignés à l'école.

En réponse au rapport de Nuffield Foundation, Jim Anderson et al. proposent que les langues parlées au Royaume-Uni autres que l'anglais soient démocratisées dans l'enseignement, au même titre que les "modern foreign languages ${ }^{21}$. L'institutionnalisation de l'enseignement d'une langue qui jusque là pouvait être enseignée dans un cadre associatif, (ou alors n'était pas du tout enseignée) engendrerait forcément des mutations d'ordre pédagogique et psycho-sociales. Pour rester dans un objectif de démocratisation de l'enseignement d'une langue, il faudrait pouvoir le proposer à tout enfant, que cette langue soit sa langue première ou une langue inconnue. Il y aurait donc des aménagements didactiques à penser afin que l'enseignement soit bénéfique pour tous.

15 Joanna McPake et Teresa Tinsley (éds), Valuing All Languages in Europe, Strasbourg / Graz : Council of Europe / European Centre for Modern Languages, 2007, http://archive.ecml.at/ $\mathrm{mtp} 2 /$ publications/Valeur-report-E.pdf, 22/10/2015.

16 Philip Baker et John Eversley, Multilingual Capital: the languages of London's schoolchildren and their relevance to economic, social and educational policies, Battlebridge publications, London, 2000.

17 Notre traduction : La diversité de Londres contribue à sa compétitivité dans le marché économique mondial d'aujourd'hui.

18 Voir les derniers résultats concernant l'inclusion des langues dans le système scolaire anglais (British Council, 2015)

19 Welsh Language Act, 1993, http://www.legislation.gov.uk/ukpga/1993/38, 22/10/2015.

20 Gaelic Language (Scotland) Act, 2005, http://www.legislation.gov.uk/asp/2005/7, 22/10/2015.

21 Jim Anderson et al., op. cit. Équivalentes aux langues vivantes étrangères en France ; il s'agit principalement du français, de l'espagnol et de l'allemand. 
En tout cas, pour ces auteurs, si ce projet doit réussir, il est nécessaire que l'école adopte une vision sympathisante du multilinguisme, au travers d'un discours positif envers les langues minoritaires. D'autres stratégies pour atteindre le plurilinguisme existent, sur critères économiques (langues les plus demandées sur le marché du travail mondial ; langues les plus parlées dans le monde), géographiques (renforcement de l'enseignement des langues de pays limitrophes), mais leur passage en revue n'est pas le propos de cet article. Dans tous les cas, avant même d'élaborer un plan de stratégie pour le plurilinguisme sur la base d'un choix de langues, il nous semble que s'impose une étape visant à changer les mentalités vis-à-vis du plurilinguisme. C'est ce que fait le projet Mother Tongue Other Tongue dont il est question ci-après.

Mother Tongue Other Tongue : prendre conscience du multilinguisme environnant

Routes into Languages est un consortium d'universités anglaises et galloises qui travaille avec les établissements scolaires primaires et secondaires pour valoriser et promouvoir l'enseignement des langues. En 2014 est lancée la première édition de Mother Tongue Other Tongue, un concours de poésie multilingue dans les écoles primaires et secondaires de Manchester. L'objectif premier de ce projet était de promouvoir l'apprentissage des langues à l'école : en valorisant les langues parlées ou apprises par les enfants, le projet avait pour ambition de faire évoluer les mentalités vis-à-vis des langues, de leurs pratiques et de leur apprentissage ${ }^{22}$.

Le concours est divisé en deux catégories d'entrées : Mother Tongue accueille des textes créés de toute pièce ou repris (chanson, poème, comptine...) en langue maternelle (sans distinction ${ }^{23}$ ); Other Tongue est dédiée aux poèmes écrits en langue étrangère enseignée dans le cadre d'une classe de "modern foreign language ". Le projet est proposé à l'ensemble des écoles de l'agglomération. Les enseignants qui s'inscrivent peuvent prendre la responsabilité du concours à l'échelle de leur établissement. La participation aux deux catégories est bien entendue envisageable.

Chaque enfant participant propose un texte (ou poème - nous reviendrons plus en détails sur ce qui est entendu par " poème ") pour la catégorie correspondante. Celui-ci est accompagné d'un texte rédigé en anglais, qui explique ses choix, les circonstances de rédaction du poème ou bien une anecdote liée au poème. Ce travail est en principe assez libre et c'est à l'enfant de choisir ce qu'il veut écrire.

22 ROUTES INTO LANGUAGES, https://www.routesintolanguages.ac.uk/index.html, 22/10/2015.

23 Cette année à Glasgow ont été évoqué l'inclusion de langues des signes ainsi que l'écriture en braille d'un poème en français. 
En parallèle de la préparation qui a lieu en classe avec l'enseignant, sont proposés pendant l'année des ateliers animés par des écrivains et artistes pour les élèves ou pour les enseignants afin d'ouvrir aux processus de création en langues, sur le travail de poésie par exemple.

Un jury composé des membres organisateurs du concours ainsi que des intervenants poètes ou artistes sélectionne ensuite les meilleurs poèmes. Une cérémonie de remise de prix est organisée. Il y a bien sûr des gagnants, mais la notion de compétition est à relativiser. Comme le disent les organisateurs du concours en Écosse qui ont remis l'an passé vingt-cinq prix pour cent entrées, "plus qu'une compétition, il s'agit d'une célébration des langues ${ }^{24}$.

Depuis son succès à Manchester en 2014, Mother Tongue Other Tongue a été repris aux quatre coins du Royaume-Uni par des organismes locaux : en Angleterre et au Pays de Galles, le projet reste sous l'égide de Routes into Languages qui dispose de sous-branches locales ; en Écosse, Mother Tongue Other Tongue est pris en charge par le SCILT (Scottish National Centre for Languages), organisme financé par le gouvernement écossais offrant du conseil aux professionnels de l'enseignement et de l'information aux familles.

Il est encore trop tôt pour savoir si Mother Tongue Other Tongue a atteint son objectif premier. En revanche, ce concours s'est présenté à chaque fois comme une véritable opportunité pour des enfants plurilingues de s'exprimer au sujet de et par le biais d'une langue qui jusque là était ignorée à l'école. C'est dans un tel contexte que vont se révéler des attitudes et des représentations vis-à-vis du multilinguisme environnant.

\section{Mother tongue other tongue : (re)trouver sa voix ${ }^{25}$}

\section{Présentation du concours en Écosse}

La première édition de Mother Tongue Other Tongue en Écosse a eu lieu en 2015 dans la ville pilote de Glasgow. Sur environ quatre-vingts écoles démarchées, vingt se sont inscrites au projet, et dix d'entre elles l'ont suivi jusqu'à son terme. Parmi ces dix écoles, neuf étaient des écoles primaires, la dixième étant une école secondaire. Une classe a participé à la catégorie Other Tongue, celle de l'école secondaire, à l'initiative de l'enseignant de français (les participations étaient donc en langue française). Toutes les autres participations entraient dans la catégorie Mother Tongue, à l'initiative d'enseignants d'EAL (anglais comme langue seconde) ou d'anglais (comme discipline). Comme nous l'avons déjà évoqué ci-dessus, le concours a reçu cent entrées et vingtcinq d'entre elles ont reçu un prix.

24 Propos recueillis lors d'une mission à Glasgow en septembre 2015 (détaillée ci-après) : « More than a competition, it is a celebration of languages".

25 Inspiré du slogan du SCILT pour Mother Tongue Other Tongue : " Find your voice ». 
Dix huit langues ont concourues : l'arabe, le mandarin, des langues nigério-congolaises (edo, igbo, yoruba, swahili), le sindebele (langue parlée au Zimbabwe et en Afrique du Sud), des langues du subcontinent indien (cingalais, ourdou), des langues d'Europe de l'Est (polonais, slovaque et géorgien), des langues d'Europe (français, italien, portugais, castillan et grec) et le scots, langue régionale ou minoritaire parlée en Écosse. L'ensemble de ces langues est représentatif de la diversité linguistique et culturelle de la ville de Glasgow, ville la plus multiculturelle en Écosse (National Records of Scotland, 2011).

Une classe de gaélique avait commencé le projet mais ne l'a pas suivi jusqu'au bout. Comment expliquer l'absence du gaélique et la présence marquée du scots, les deux langues régionales ou minoritaires en Écosse ? D'après les organisateurs ${ }^{26}$, il est difficile de sensibiliser le public d'apprenants du gaélique car cette langue bénéficie de soutiens indépendants privilégiés et les défenseurs de cette langue tendent à s'écarter des mouvements en faveur de la promotion des langues en général. Les organisateurs aimeraient cependant voir cette langue s'ajouter au panel de langues représentées.

Le scots, pratiqué sur le territoire de l'Écosse, a longtemps été perçu de manière négative mais les attitudes envers cette langue sont en train de changer et l'école l'intègre dans son programme. En revanche, le scots n'est pas enseigné dans un cours de langue, mais il fait partie des "Scottish studies ", il est ainsi intégré dans les cours de littérature comme "spécificité régionale " (Conseil de l'Europe, 2014) ${ }^{27}$. Les entrées en scots ont donc été motivées par des enseignants d'anglais (littérature). ${ }^{28}$

Les autres langues représentées par la catégorie Mother Tongue ne bénéficient pas d'un enseignement à l'école. Certaines sont cependant enseignées dans des "community language schools" en marge du système scolaire.

La configuration linguistique et urbaine de Glasgow en fait un terrain tout à fait intéressant pour nos recherches qui tentent de considérer le multilinguisme dans sa globalité : deux langues régionales ou minoritaires, qui ne bénéficient ni du même statut ni du même degré de promotion et qui n'ont pas la même image auprès des écossais, sont en contact avec des langues allochtones d'immigration récente (polonais) ou plus ancienne (ourdou).

26 Entretien réalisé le 06/03/2015 à Glasgow.

27 Propos confirmés lors de l'entretien avec le SCILT à Glasgow le 06/03/15.

28 Notons que l'anthologie des poèmes lauréats de 2015 sépare les poèmes en scots des deux autres catégories de concours. Les organisateurs expliquent ce choix par une volonté de promouvoir le scots, en nominant l'ensemble des poèmes présentés dans cette langue. Les organisateurs de l'édition 2016 sont indécis quant à la catégorie dans laquelle le scots doit entrer cette année (Mother Tongue ou Other Tongue?). 


\section{Le poème comme forme d'expression}

Mother Tongue Other Tongue a choisi la poésie comme moyen d'expression, sans pour autant définir ce champ. Le projet ne s'inscrit pas dans un programme scolaire précis. En revanche, le SCILT a diffusé sur son site une liste de compétences et d'approches scolaires qui s'en trouveraient favorisées, parmi lesquelles une approche créative de l'apprentissage des langues, un travail sur des textes au programme (la poésie scots et la chanson gaélique sont données comme exemple) et le travail d'expression écrite. Il s'agit avant tout de donner aux élèves une opportunité de s'exprimer sans contrainte. Libre cours à l'enseignant de présenter le travail de poésie comme il le sent.

En septembre 2015, nous nous sommes rendue à Glasgow dans le cadre d'un atelier proposé aux enseignants participant à l'édition 2016. Mené par une poétesse écossaise, le but de cet atelier était d'activer le potentiel de créativité dont nous disposons tous par le biais de l'écriture. À l'issue, les enseignants sont libres de puiser dans cette expérience les éléments à réitérer dans leur classe.

L'étude de l'anthologie de l'édition 2015 montre que des contraintes de rédaction ont pu être imposées par certains enseignants ${ }^{29}$. C'est le cas des entrées pour le scots qui suivent un modèle de rédaction, et dont le texte explicatif semble répondre à une matrice de questions. D'autres enseignants semblent beaucoup plus ouverts à la forme que doit prendre la prose de l'enfant. Par exemple, cette année, certains ont évoqué l'idée de laisser les enfants s'exprimer par le rap. Le SCILT a répondu de manière positive à cette idée et a proposé de développer en conséquence d'autres moyens de diffusion (comme la vidéo) en parallèle de l'écrit qui reste indispensable pour conserver une forme d'expression commune.

Le but pour les organisateurs n'est donc pas la poésie en soi mais plutôt de mettre à disposition le maximum d'outils pour que les enfants se sentent libres de s'exprimer au sujet de et dans leur langue. L'analyse sociolinguistique de cette forme de création nécessitera très certainement des outils faisant appel à l'interdisciplinarité. Nous ne cachons pas l'attrait et la stimulation qu'a suscités l'aspect artistique de Mother Tongue Other Tongue.

\section{Analyse des premiers poèmes - Glasgow, édition 2015}

Afin d'illustrer l'anthologie de l'édition 2015 de Glasgow, voici un extrait présentant le poème d'une jeune fille qui alterne habilement l'anglais et l'ourdou, accompagné de son texte explicatif rédigé en anglais.

29 SCOTLAnd'S national CENTRE FOR LANgUages (SCILT), Mother Tongue Other Tongue 20142015, Anthology, 2015, http://www.scilt.org.uk/Portals/24/Library/MTOT/Celebration \%20 event/mtot \%20anthology \%20final.pdf, 22/10/2015. 


\section{Bari}

I enjoyed the poetry workshop with Ken Cockburn. I liked hearing the poem in German. It was fun because I am not used to hearing poems in other languages. So, that inspired me to write in Urdu.

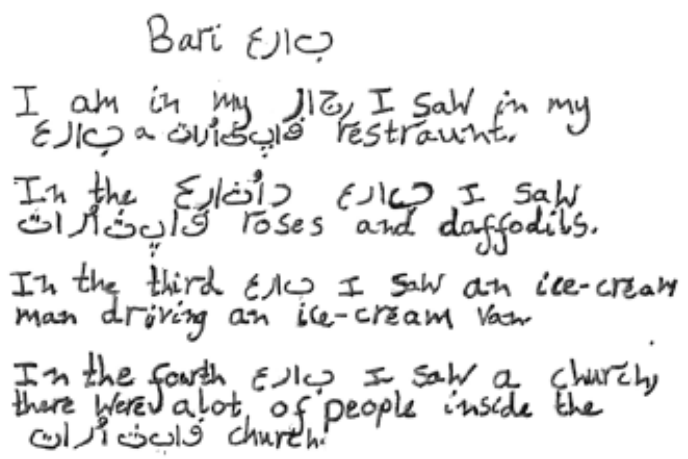

My poem is about what I see when I look out the windows of my house. It was easier writing in Urdu because that is my first language. I wrote in Urdu and in English because I thought it would be fun to do and fun for people to hear. Everyone in class said, "It is so cool that you know another language!" They were impressed that I could read and write in another language because they can't do this.

In figth Ele? I saw a lisid,

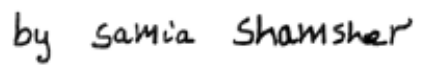

My favourite part is "roses and daffodils" because it makes people think about and imagine summer, all the pretty stuff and all the beauty of summer in Scotland. If someone from another country reads this, I hope they would think Scotland is a good place to spend their summer. I wanted people to imagine the beautiful smelling daffodils and seeing lots of exciting things and meeting new people.

\section{Samia Shamsher,}

P7b, King's Park Primary School, (Urdu)

Figure 1 : Extrait de l'anthologie de Mother Tongue Other Tongue, (SCILT, 2015, p. 19).

L'étude de cette anthologie a permis de mettre en avant certaines caractéristiques communes. Tout d'abord, nombreuses sont les entrées qui témoignent d'un sentiment d'appartenance identitaire ou d'un attachement aux territoires d'origine et d'adoption (à travers le poème ou le texte explicatif). Dans l'extrait ci-dessus, l'enfant exprime son désir de rendre compte de la beauté de l'Écosse en été, de donner l'envie au lecteur étranger "d'y passer son été ». Le territoire adopté est à l'honneur. Dans un autre poème, un enfant a choisi de présenter l'hymne national de Pologne comme entrée de compétition, symbole fort d'un attachement au territoire d'origine (SCILT, 2015, p. 12) ; une autre jeune fille présente un poème écrit en version bilingue anglais-polonais. Pour elle, «dom » (titre du poème en polonais - signifiant « la maison » en français) fait référence à la Pologne. Elle évoque dans son argumentaire des souvenirs de la maison qu'elle a connue plus jeune en Pologne (SCILT, 2015, p. 17).

C'est là la deuxième caractéristique commune : il semblerait que le recours à la langue d'héritage invite aux souvenirs de famille et à l'évocation d'un vécu dans le territoire d'origine. Une jeune fille a choisi de reprendre une chanson 
que sa grand-mère lui chantait plus jeune et qu'elle continue de chanter avec ses parents en polonais : ce choix montre l'importance affective et familiale qu'occupe la langue maternelle (SCILT, 2015, p. 14).

Certains enfants ont choisi de profiter de cette occasion de s'exprimer en langue maternelle pour faire connaitre la culture, l'histoire ou l'actualité marquant leurs origines, tout en reliant ce contexte à leur vie actuelle à Glasgow. C'est ce qu'ont choisi de faire deux jeunes sœurs Iraqi, en racontant les raisons pour lesquelles leurs parents ont décidé de fuir le pays (SCILT, 2015, p. 18) :
"We enjoyed writing our poem. We wrote this poem about Iraq. We wanted people to know how dangerous Baghdad was when we lived there. It is even more dangerous in Mosul, so my family moved from Mosul to Baghdad. There is lots of fighting in Mosul and in Baghdad, so we are happy in Glasgow with our parents and brother, but we miss our other family in Iraq. $»^{30}$

D'un point de vue linguistique, les poèmes ont mis en avant des compétences variables en langues mais une certaine créativité et ingéniosité à les utiliser. Le cadre non normatif du concours signifie que l'enfant est libre de créer un poème à partir d'une langue qu'il maîtrise plus ou moins bien. Les poèmes créés de toute pièce témoignent de l'inventivité dont font preuve les enfants, notamment pour gérer certaines lacunes. En lisant le poème en alternance codique (anglais/andou) plus haut, on peut se demander si l'enfant a utilisé cette technique pour pallier à des difficultés, alliant pour le meilleur ses compétences dans chacune des deux langues. Or, le texte explicatif nous indique plutôt qu'il s'agit d'un choix esthétique. On peut d'ailleurs remarquer la régularité de l'alternance : le poème est construit sur une base syntaxique de l'anglais, l'enfant insère des éléments lexicaux en ourdou (syntagmes nominaux). D'autres auteurs qui ont choisi d'écrire en deux langues précisent leurs difficultés linguistiques et le recours conséquent à une aide familiale, comme cette auteure qui a écrit le poème bilingue anglais-polonais "Dom » : "This is the first poem that I made. I wrote a poem in English. I found it hard to write in Polish. My brother helped me spell the words in Polish. He knows most of the words. ${ }^{31}$.

À travers les propos des textes explicatifs, on note une certaine conscience métalinguistique de la part de ces enfants plurilingues. La jeune fille, dont

30 Notre traduction : Nous avons aimé écrire notre poème. Nous avons écrit ce poème à propos de l'Irak. Nous voulions que les gens sachent à quel point c'était dangereux à Bagdad lorsque nous y vivions. C'est encore plus dangereux à Mossoul, alors ma famille a déménagé de Mossoul à Bagdad. Il y a beaucoup d'affrontements à Mossoul et Bagdad, nous sommes donc heureuses à Glasgow avec nos parents et notre frère, mais le reste de notre famille (ou notre autre famille) nous manque en Irak.

31 Notre traduction : Ceci est le premier poème que j'ai écrit. J'ai écrit un poème en anglais. J'ai trouvé cela difficile d'écrire en polonais. Mon frère m’a aidé à écrire les mots en polonais. Il connait la plupart des mots. 
le poème fait figure d'illustration plus haut, montre une grande ouverture d'esprit vis-à-vis des langues, une capacité de réflexion sur les langues : elle a " aimé " " entendre " un poème en allemand, elle a trouvé l'expérience globale " amusante » car elle n'a pas l'habitude d'" entendre » des poèmes dans "d'autres langues" (notre traduction). Son texte met aussi en avant des attitudes extrêmement positives de la part de ses camarades de classe vis-à-vis de ses compétences linguistiques, ce qui semble être une des réactions souhaitées de la part des organisateurs pour réaliser le premier objectif du concours.

D'autres enfants déclarent dans leur texte explicatif qu'ils se sentent plus créatifs, plus libres dans une langue que dans l'autre. C'est le cas de certaines entrées en scots (rappelons toutefois la forme répétitive de ces entrées qui laisse penser qu'une sorte de matrice a été imposée dans la rédaction des poèmes et des textes explicatifs) :

«I don't think they go well together in English but in Scots they do. Writing in Scots was fun because you could write about what you wanted. "32 $^{32}$

"I used Scots words because I thought it would open up my imagination and be much more effective to the reader. "33 $^{33}$

"I liked writing in Scots because it was much funnier than writing in English.

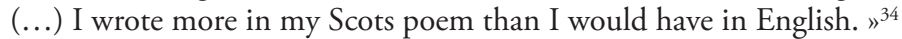

En revanche, un poème laisse transparaitre des attitudes encore négatives vis-à-vis du scots : "It was a bit weird writing in Scots because I don't normally speak in Scots. Sometimes my dad uses Scots words and I give him a row! „" ${ }^{35}$ On peut aussi interpréter cette réaction comme celle de l'enfant en phase de dénigrement du bilinguisme familial.

Enfin, on note l'importance de l'implication familiale dans ce projet d'écriture. Les enfants évoquent dans leur texte explicatif la participation normative d'un parent dans le projet, aidant à combler certaines faiblesses linguistiques : "My poem is about mothers. My mum helped me to write it. $"^{36} ;$ "I wrote the poem and then mum checked it for me $»^{37}$. Mise à part cette relecture des parents, aucune lecture à visée normative n'est fournie par le jury (dont les membres ne présentent pas forcément les compétences linguistiques adéquates). Le parent participe ainsi à la fois en soutenant l'enfant dans sa

32 Notre traduction : je ne trouve pas que [ces mots] vont bien ensemble en anglais mais en scots, si. Écrire en scots était amusant parce que l'on pouvait écrire à propos de ce que l'on voulait.

33 Notre traduction : J'ai utilisé des mots scots parce que je pensais que cela pourrait développer mon imagination et avoir beaucoup plus d'impact sur le lecteur.

34 Notre traduction : J'ai aimé écrire en scots car c'était beaucoup plus drôle qu'écrire en anglais (...) J'ai écrit plus dans mon poème en scots que je ne l'aurais fait en anglais.

35 Notre traduction : C'était bizarre d'écrire en scots parce que normalement je ne parle pas en scots. Parfois mon père utilise des mots scots et alors je le fâche!

36 Notre traduction : Mon poème est à propos des mères. Ma mère m’a aidé à l'écrire.

37 Notre traduction : J'ai écrit le poème, puis ma mère l'a corrigé pour moi. 
démarche et en jouant le rôle de jury normalisateur, rôle qu'il n'a pas forcément l'habitude de jouer, puisqu'aucun renseignement n'est donné quant à l'apprentissage formel ou informel que l'enfant suit dans sa langue maternelle. Seule une enquête complémentaire permettra de le déterminer. C'est là l'objet de notre prochain chapitre.

\section{Éléments de méthodologie de l'enquête en cours - Édition 2016}

Une enquête de terrain est envisagée de manière imminente afin de suivre le travail évolutif des enfants participant à l'édition 2016 du concours en Écosse. Nous nous rendrons dans des écoles primaires et secondaires situées à Glasgow, Édimbourg, Dundee et Inverness. Il s'agira de suivre l'évolution du projet Mother Tongue Other Tongue et de voir comment celui-ci influence les représentations et attitudes des participants vis-à-vis de la diversité linguistique et de leur plurilinguisme. Pour cela, nous procèderons à l'observation des ateliers et cours menés autour de l'élaboration des poèmes, et nous complèterons celle-ci d'entretiens oraux semi-guidés avec les enseignants et les enfants au fil du projet.

D'autres méthodes d'investigation sont prévues une fois les poèmes finaux rendus : l'observation du jury (les critères de sélection étant encore flous, nous aimerions pouvoir étudier la manière dont les organisateurs gèrent l'aspect encore aléatoire et expérimental du projet) ; l'observation de la remise des prix (où tous les acteurs seront présents, organisateurs, enseignants, enfants, parents) ; enfin, une analyse linguistique et fonctionnelle (en cas d'alternance des langues par exemple) des poèmes produits cette année appuyée par tout le travail effectué au préalable (éditions 2015 et 2016). Dans le cadre de cet article, nous nous limitons à la présentation des méthodes d'investigation précédant la remise des productions finales.

Observer la classe en pleine élaboration des projets Mother Tongue Other Tongue permettra dans un premier temps de se familiariser avec le groupe. Aussi, lors de cette observation, nous pourrons être amenée à noter certains points qui pourront ensuite être éclairés lors d'entretiens. L'étude du discours de l'enseignant et des interactions avec les enfants révèlera des attitudes des deux parties vis-à-vis du multilinguisme, des différentes langues en présence et de la créativité en langue autre que l'anglais. Une observation sur plusieurs séances accompagnée d'entretiens avec enseignants et élèves nous permettra en outre de mesurer l'influence de l'enseignant tant sur le processus de création des enfants et sur leurs productions que sur leurs attitudes et représentations linguistiques.

Après avoir recueilli certains éléments permettant de contextualiser la classe, les entretiens oraux semi-guidés auprès des enseignants auront pour but de faire émerger de leurs discours des représentations et attitudes vis-à-vis du multilinguisme britannique, de la compétence plurilingue, et des langues 
présentes dans la classe. Pour ce faire, nous les inviterons à expliquer les raisons ayant motivé leur choix de participation, la façon dont Mother Tongue Other Tongue s'intègre dans une démarche professionnelle personnelle; nous leur demanderons de s'exprimer au sujet d'éventuelles attentes vis-à-vis du projet, des approches pédagogiques et philosophiques adoptées et de préciser le temps qu'ils envisagent de consacrer à Mother Tongue Other Tongue. À l'approche du rendu des poèmes, un autre entretien viendra leur demander de dresser un bilan de leur expérience ; ils pourront à ce moment évoquer des surprises, des évolutions dans les comportements ou attitudes des enfants, et il leur sera demandé ce que l'expérience globale leur a apporté d'un point de vue personnel. L'analyse ultérieure des discours produits viendra rendre compte de leurs représentations et attitudes et des possibles évolutions de celles-ci au cours de Mother Tongue Other Tongue.

Le guide d'entretien auprès des élèves sera davantage centré sur le poème choisi par l'enfant. En partant de ce support et toujours de manière semiguidée, l'objectif de ces entretiens sera de faire émerger des discours produits les représentations et attitudes de l'enfant vis-à-vis du multilinguisme environnant, de la/des langue(s) dont il dispose dans son répertoire linguistique. Ainsi, il lui sera demandé de s'exprimer au sujet du processus de création suivi, de prendre du recul sur le poème produit ou en cours de production, d'expliquer ce qui relie le poème à son auteur, de s'exprimer au sujet de la relation entretenue avec la langue utilisée, d'évaluer le rapport entre cette langue et l'anglais, et entre cette langue et Glasgow. À l'approche du rendu des poèmes, un deuxième entretien viendra demander à l'enfant de dresser un bilan de l'expérience, d'expliquer les différentes étapes personnelles traversées durant ce travail, d'évoquer certaines éventuelles réactions, et de commenter l'argumentaire accompagnant le poème.

Pour faire émerger des représentations du discours de l'enfant, nous l'inciterons à s'exprimer de manière assez libre au sujet de son vécu des langues. En racontant des expériences personnelles ou l'histoire linguistique de la famille, l'enfant construit des récits langagiers ${ }^{38}$. Ces récits sont traités en termes narratifs et à l'aide des outils de l'analyse du discours. Nous nous intéresserons tant au contenu de ces énoncés pour déceler des représentations qu'à l'organisation de cet énoncé, témoignant de la façon dont l'élève gère son vécu et reconstruit sa vision des choses dans le discours et en fait un objet discursif. Plusieurs entretiens permettront de voir l'évolution des représentations.

La comparaison entre discours de l'enseignant et discours de l'élève, tout en tenant compte de l'évolution observée pendant les ateliers, nous permettra d'évaluer l'influence du discours de l'enseignant sur celui de l'élève, ce que l'analyse seule des poèmes ne peut mesurer entièrement. 
L'enquête entend ainsi vérifier l'impact de l'école sur les représentations de l'enfant. Nous formulons l'hypothèse suivante, à savoir que l'enfant est le lieu de contact entre deux conceptions de la langue : l'école et la famille. L'ensemble des représentations de l'enfant est sous l'influence du cadre formel d'apprentissage dans lequel il est inscrit et du cadre informel que constitue sa famille et son entourage proche ${ }^{39}$. Mother Tongue Other Tongue, en offrant une opportunité aux enfants de s'exprimer au sujet de et dans leur langue maternelle, intervient dans cette dichotomie qui régissait jusqu’alors les représentations liées aux langues.

Dans un État marqué par une telle dichotomie linguistique (monolinguisme assumé versus multilinguisme avéré) et par un enseignement des langues peu performant et peu demandé, il semble évident qu'avant de modifier les cursus d'enseignement, une vision plus positive et ouverte sur le plurilinguisme soit nécessaire au sein de la société. Utiliser le multilinguisme latent comme une ressource tant pour favoriser une prise de conscience des avantages du plurilinguisme que pour en atteindre les objectifs de compétences semble être une solution viable.

Cette démarche écologique (au sens de Louis-Jean Calvet ${ }^{40}$ ) est néanmoins tout à fait contemporaine et anticipatrice d'un avenir de plus en plus métissé et assumé en tant que tel. Elle favorise un décloisonnement des frontières entre langues au niveau des représentations collectives. Notre démarche personnelle se trouve en adéquation avec celle-ci : considérer toutes les langues présentes sur un même plan pour comprendre le multilinguisme au Royaume-Uni comme un système global et participatif.

Pour répondre à cet objectif de recherche doctorale, précisons qu'outre le champ de l'éducation, d'autres niveaux de lecture du multilinguisme sont pris en compte, tels que les discours des politiques et juristes. Ceux-ci sont marqués par des usages variés d'un nombre foisonnant de notions qui catégorisent les langues. S'ils présentent des enjeux différents, les différents niveaux de lecture du multilinguisme (la politique, le droit, l'école, la presse, la société) sont tout de même inter-reliés et peuvent s'influencer mutuellement. La dynamique actuelle de promotion du plurilinguisme au Royaume-Uni par le biais d'une valorisation du multilinguisme présent mérite d'être étudiée avec attention, car elle pourrait influencer bien plus que la sphère de l'éducation. En effet, nous serons amenée à voir si cette dynamique peut avoir des répercussions au niveau juridique et politique de l'État, si elle affectera le recours à la catégorisation des langues.

Malika Pedley IKER UMR 5478

Université Bordeaux Montaigne malika.pedley@universite-bordeaux-montaigne.fr

39 Hypothèse au cœur du mémoire de Masters soutenu en juin 2012.

40 Louis-Jean Calvet, Pour une écologie des langues du monde, Plon, Paris, 1999. 


\section{Résumé}

L'article pose les jalons d'une enquête menée en Écosse entre 2015 et 2016. Mother Tongue Other Tongue est un concours de poésie multilingue inscrit dans une démarche de promotion de l'apprentissage des langues à l'école. En exposant et en valorisant la réalité multilingue locale, toutes langues confondues, nous faisions l'hypothèse que ce concours permet aux enfants d'adopter une approche plus positive et inclusive des langues en situation minoritaire ; une vision nécessaire pour l'apprentissage des langues dans un monde où la mobilité est un phénomène de plus en plus courant et de plus en plus complexe.

\section{Mots-clés}

Politiques linguistiques, éducation, plurilinguisme, multilinguisme, écriture créative.

\section{Abstract}

This paper precedes fieldwork conducted in Scotland between 2015 and 2016. Mother Tongue Other Tongue is a multilingual poetry competition which aims to promote language learning at school. By exposing and valuing local multilingual reality, all languages included, our hypothesis was that this competition enables children to adopt a more positive and inclusive approach to languages in a minority situation - a necessary vision for language learning, in step with a world where mobility as a phenomenon becomes more and more commonplace and more and more complex.

\section{Keywords}

Language policies, education, plurilingualism, multilingualism, creative writing. 